Corrigendum: Ulcerative colitis-risk loci on chromosomes 1p36 and 12q15 found by genome-wide association study

Mark S Silverberg, Judy H Cho, John D Rioux, Dermot P B McGovern, Jing Wu, Vito Annese, Jean-Paul Achkar, Philippe Goyette, Regan Scott, Wei Xu, M Michael Barmada, Lambertus Klei, Mark J Daly, Clara Abraham, Theodore M Bayless, Fabrizio Bossa, Anne M Griffiths, Andrew F Ippoliti, Raymond G Lahaie, Anna Latiano, Pierre Paré, Deborah D Proctor, Miguel D Regueiro, A Hillary Steinhart, Stephan R Targan, L Philip Schumm, Emily O Kistner, Annette T Lee, Peter K Gregersen, Jerome I Rotter, Steven R Brant, Kent D Taylor, Kathryn Roeder \& Richard H Duerr

Nat. Genet. 41, 216-220 (2009), published online 4 January 2009; corrected after print 28 April 2009

In the first paragraph of the second column on the third page, rs11209026 A allele was incorrectly listed as rs111209026 A allele. The error has been corrected in the HTML and PDF versions of the article.

Corrigendum: Loss-of-function mutations of an inhibitory upstream ORF in the human hairless transcript cause Marie Unna hereditary hypotrichosis

Yaran Wen, Yang Liu, Yiming Xu, Yiwei Zhao, Rui Hua, Kaibo Wang, Miao Sun, Yuanhong Li, Sen Yang, Xue-Jun Zhang, Roland Kruse, Sven Cichon, Regina C Betz, Markus M Nöthen, Maurice A M van Steensel, Michel van Geel, Peter M Steijlen, Daniel Hohl, Marcel Huber, Giles S Dunnill, Cameron Kennedy, Andrew Messenger, Colin S Munro, Alessandro Terrinoni, Alain Hovnanian, Christine Bodemer, Yves de Prost, Amy S Paller, Alan D Irvine, Rod Sinclair, Jack Green, Dandan Shang, Qing Liu, Yang Luo, Li Jiang, Hong-Duo Chen, Wilson H-Y Lo, W H Irwin McLean, Chun-Di He \& Xue Zhang Nat. Genet. 41, 228-233 (2009), published online 4 January 2009; corrected after print 28 April 2009

The affiliation of the 24th author, Alessandro Terrinoni, was listed incorrectly. It should read IDI-IRCCS Biochemistry Laboratory c/o Univ. Tor Vergata, 00133 Rome, Italy. The error has been corrected in the HTML and PDF versions of this article.

\title{
Addendum: Deep surveying of alternative splicing complexity in the human
} transcriptome by high-throughput sequencing

Qun Pan, Ofer Shai, Leo J Lee, Brendan J Frey \& Benjamin J Blencowe

Nat. Genet. 40, 1413-1415 (2008), published online 2 November 2008; addendum published after print 28 April 2009

The GEO accession number for the mRNA-Seq datasets is GSE13652.

Corrigendum: Genome-wide association of early-onset myocardial infarction with single nucleotide polymorphisms and copy number variants

Myocardial Infarction Genetics Consortium

Nat. Genet. 41, 334-341 (2009); published online 8 February 2009; corrected after print 27 May 2009

In the version of this article initially published, the names of four co-authors (Christopher W Knouff, Dawn M Waterworth, Max C Walker, Vincent Mooser) were omitted from the author list. The error has been corrected in the HTML and PDF versions of the article. 